\title{
Upregulation of the expression of Wnt5a promotes the proliferation of pancreatic cancer cells in vitro and in a nude mouse model
}

\author{
HAIJI BO ${ }^{1}, \mathrm{LI} \mathrm{GAO}^{2}$, YING $\mathrm{CHEN}^{2}$, JING ZHANG ${ }^{2}$ and MINGHUA ZHU ${ }^{2}$ \\ ${ }^{1}$ Department of Pathology, No. 455 Hospital of PLA, Shanghai 200052; ${ }^{2}$ Department of Pathology, \\ Changhai Hospital, The Second Military Medical University, Shanghai 200433, P.R. China
}

Received January 5, 2015; Accepted October 14, 2015

DOI: $10.3892 / \mathrm{mmr} .2015 .4642$

\begin{abstract}
Wnt proteins are a group of secreted signaling proteins, which function to regulate cell fate and pattern formation during embryogenesis. Altered expression of Wnt5a has been implicated in human carcinogenesis and tumor progression. A previous study identified that Wnt5a is overexpressed in human pancreatic cancer tissues, and that upregulated expression of Wnt5a promotes tumor cell migration and invasion. The present study investigated the role of Wnt5a in pancreatic cancer cell proliferation in vitro and in an orthotopic nude mouse model. Wnt5a cDNA or small interfering RNA were stably transfected into pancreatic cancer cells to assess cell proliferation-associated behaviors, including cell viability, colony formation and apoptosis in vitro, as well as tumor cell growth in an orthotopic nude mouse model. Western blot analysis was used to analyze the expression of Wnt signaling molecules. The data showed that upregulation of the expression of Wnt5a significantly promoted proliferation of the human pancreatic cells, but inhibited tumor cell apoptosis in vitro and promoted tumor growth in an orthotopic nude mouse model. By contrast, knockdown of the expression of Wnt5a inhibited cell growth and promoted apoptosis of the pancreatic cancer cells. The data also revealed that $\beta$-catenin mediated the effects of Wnt5a on the regulation of pancreatic cancer cell apoptosis in vitro. These results suggested that Wnt5a is involved in the modulation of pancreatic cancer cell proliferation, and that Wnt5a may be a potential target for pancreatic cancer therapy.
\end{abstract}

Correspondence to: Dr Minghua Zhu, Department of Pathology, Changhai Hospital, The Second Military Medical University, 168 Changhai Road, Shanghai 200433, P.R. China

E-mail: minghuazhu2000@163.com

Key words: pancreatic cancer, Wnt5a, tumor growth, cell viability, apoptosis, animal model

\section{Introduction}

Pancreatic cancer is a significant worldwide health problem, with an estimated 277,000 new cases, and a cancer-associated mortality rate of 266,000 annually worldwide (1). The majority of patients with pancreatic cancer are diagnosed at advanced stages of the disease (2), at which tumor tissues are surgically unresectable and chemotherapy is mostly ineffective, leading to a 5-year survival rate of $\sim 4 \%$ (3). Thus, there is a significant and urgent requirement to elucidate the molecular mechanisms that mediate pancreatic cancer development and progression, in order to effectively control and prevent this life-threatening disease. The development of pancreatic cancer, as with other types of cancer in the gastrointestinal tract, involves multiple genetic alterations, including oncogene activation and tumor suppressor gene dysfunction. Over the past three decades, a large body of evidence has accumulated regarding the molecular alterations associated with the development of pancreatic cancer (4-6). These disparate observations have coalesced somewhat into an improved understanding of this disease, however, further investigation is required to provide early cancer identification and novel treatment strategies for pancreatic cancer.

The present study focused on Wnt signaling proteins in pancreatic cancer. Wnt consists of a large family of secreted lipid-modified glycoproteins, which regulate numerous processes in animal development (7) and normal cell processes, including cell growth, differentiation, apoptosis, migration and cell polarity (8). Each member of the Wnt family of proteins exhibits unique expression patterns and has distinct functions in embryonic development and normal cell homeostasis (9). Based on their ability to induce transformation of the mouse mammary epithelial cell line, Wnt proteins are divided into two functional groups, canonical and noncanonical Wnt (10). Wnt5a is classified as a member of the noncanonical Wnt family, is crucial for embryonic development and has been implicated in several human diseases (11-13). Previous studies have demonstrated that Wnt5a is important in the progression of various types of human cancer (14-16); however, the particular signaling and physiological function of the Wnt5a protein in pancreatic cancer remains to be elucidated. Our previous study identified that Wnt5a protein is expressed at high levels in human pancreatic cancer tissues, and that upregulation of 
Wnt5a promotes epithelial-to-mesenchymal transition and metastasis of pancreatic cancer cells (17). On the basis of these data, the aim of the present study was to characterize the role of Wnt5a in the proliferation of pancreatic cancer cells, and to elucidate the signaling events of the Wnt5a protein.

\section{Materials and methods}

Cell lines and culture. Human PANC-1 and BXPC-3 pancreatic cancer cell lines were obtained from the Institute of Cellular Research, Chinese Academy of Science (Shanghai, China) and routinely cultured in RPMI 1640 medium supplemented with $10 \%$ fetal bovine serum, $100 \mathrm{U} / \mathrm{ml}$ penicillin and $100 \mathrm{U} / \mathrm{ml}$ streptomycin (all from Invitrogen; Thermo Fisher Scientific, Inc., Waltham, MA, USA) at $37^{\circ} \mathrm{C}$ in $5 \% \mathrm{CO}_{2}$. The medium was replaced every third day.

Gene transfection into pancreatic cancer cells. Plasmids carrying Wnt5a cDNA were constructed by the subcloning of Wnt5a cDNA into a pcDNA3.1 vector (Invitrogen; Thermo Fisher Scientific, Inc.) with double restriction enzyme (XbaI and BamHI; Invitrogen; Thermo Fisher Scientific, Inc.) digestion. Subsequently, the pcDNA3.1-WNT5A vector or empty pcDNA3.1 vector was transfected into the PANC-1 and BXPC-3 cells (60-80\% confluent) using Lipofectamine 2000 (Invitrogen; Thermo Fisher Scientific, Inc.), according to the manufacturer's protocol. To establish cells stably overexpressing Wnt5a, the cells were selected in G418-containing medium $(1,000 \mu \mathrm{l} / \mathrm{ml}$ for PANC-1 and $400 \mu \mathrm{l} / \mathrm{ml}$ for BXPC-3 cells; Invitrogen; Thermo Fisher Scientific, Inc.). Following 2-3 weeks of $\mathrm{G} 418$ selection $\left(37^{\circ} \mathrm{C}\right.$ in $\left.5 \% \mathrm{CO}_{2}\right)$, single cell clones were isolated, expanded and screened for the expression of Wnt5a using immunoblot analysis.

To knock down endogenous expression of Wnt5a in the pancreatic cancer cells, the present study utilized small interfering RNA (siRNA) technology, using the target sequence, 5'-GTTTTGGCCACTGACTGA-3'. The Wnt5a siRNA expression cassette (FuNeng Biotech Co., Ltd., Guangzhou, China) was subcloned into a pcDNA6.2 vector (Invitrogen; Thermo Fisher Scientifc, Inc.), following which the pcDNA6.2-WNT5A-siRNA vector or the pcDNA6.2 control vector was transfected into the PANC-1 and BXPC-3 cells (60-80\% confluent) using Lipofectamine 2000, according to the manufacturer's protocol. Blasticidin (Merck Millipore $\mathrm{GmbH}$, Darmstadt, Germany) was used to screen resistant cell clones ( $4 \mu \mathrm{g} / \mathrm{ml}$ for PANC-1 and $2 \mu \mathrm{g} / \mathrm{ml}$ for BXPC-3 cells). Stable clones were confirmed by immunoblot analysis.

In addition, $\beta$-catenin and control siRNA were obtained from GenePharma Company (Shanghai, China) to knock down the expression of $\beta$-catenin. The $\beta$-catenin or control siRNA (20 $\mathrm{nM}$ ) was then transfected into the pancreatic cancer cells and, after $24 \mathrm{~h}$ culture at $37^{\circ} \mathrm{C}$ in $5 \% \mathrm{CO}_{2}, 200 \mathrm{ng}$ of recombinant human Wnt5a peptide (Abcam) was added to the cell culture. The expression levels of nuclear $\beta$-catenin were detected by immunoblot analysis.

Western blot analysis. The cells were lysed in lysis buffer (Invitrogen; Thermo Fisher Scientific, Inc.), and the proteins were separated on $10 \%$ SDS-PAGE (Shenggong Biotech Co., Ltd., Shanghai, China) prior to being transferred onto
$0.45 \mu \mathrm{m}$ pore polyvinylidene difluoride membranes (Bio-Rad Laboratories, Inc., Hercules, CA, USA). The membranes were probed with the following primary antibodies: Rabbit polyclonal anti-Wnt5a (1:1,000; cat. no. ab72583; Abcam, Cambridge, MA, USA), mouse monoclonal anti-glyceraldehyde-3-phosphate dehydrogenase (GAPDH; 1:3,000; cat. no. KC-5G4; Kangchen Biotech, Inc., Shanghai, China), rabbit monoclonal anti- $\beta$-catenin $(1: 1,000$; cat. no. ab32572; Abcam) and rabbit polyclonal anti-lamin A/C (1:1,000; cat. no. sc-20681; Santa Cruz Biotechnology, Inc., Dallas, TX, USA). Subsequently, the membrane was washed once in $1 \mathrm{X}$ TBS (1X Tris-buffered saline: 50 mM Tris- $\mathrm{HCl}, \mathrm{pH}$ 7.4; $150 \mathrm{mM}$ $\mathrm{NaCl}$; Shenggong Biotech Co., Ltd.) and three times in $1 \mathrm{X}$ TBS-T buffer (1X TBS containing 0.1\% Tween 20; Shenggong Biotech Co., Ltd.). The goat polyclonal anti-rabbit (1:5,000; cat. no. ab6720; Abcam) or goat monoclonal anti mouse (1:5,000; cat. no.ab197767; Abcam) secondary antibodies were added in blocking solution (3\% nonfat dry milk in TBS-T buffer) and the membranes were shaken for $2 \mathrm{~h}$ at room temperature. Reactive proteins were detected using enhanced chemiluminescence (GE Healthcare Bio-Sciences, Pittsburgh, PA, USA). The intensity of each band was measured by densitometric analysis using the Quantity One software (Bio-Rad Laboratories, Inc.).

Cell viability assay. The stable PANC-1 and BXPC-3 cells exhibiting Wnt5a overexpression or knockdown were seeded into a 96-well plate at $1 \times 10^{3}$ cells/well and maintained in G418-conditioned medium, with eight replicate wells used for each group. After 24, 48, 72, 96, 120, 144 and $168 \mathrm{~h}$ of cell culture at $37^{\circ} \mathrm{C}$ in $5 \% \mathrm{CO}_{2}, 400 \mu \mathrm{g} / \mathrm{ml}$ of methylthiazolyldiphenyl-tetrazolium bromide (MTT; Sigma-Aldrich, St. Louis, MO, USA) was added to each well, and the cells were incubated for an additional $4 \mathrm{~h}$ at $37^{\circ} \mathrm{C}$. Subsequently, solubilization was performed using $150 \mu \mathrm{l}$ dimethyl sulfoxide (DMSO; Tiangen Biotech Co., Ltd., Beijing, China) and incubated at $37^{\circ} \mathrm{C}$ in $5 \% \mathrm{CO}_{2}$ for $20 \mathrm{~min}$. The optical density was then measured using a spectrophotometer (Ultrospec K; Biochrom, Ltd., Berlin, Germany) at a wavelength of $570 \mathrm{~nm}$. The optical density values of each experiment were plotted to determine the cell viability curve. The experiment was repeated three times.

Tumor cell colony formation assay. For the clonogenic assay, the BXPC-3 and PANC-1 cells exhibiting Wnt5a overexpression or knockdown were plated at a density of 1,000 viable cells/well in six-well plates and cultured for 14 days in a $37^{\circ} \mathrm{C}$ incubator. The cells were then washed with phosphate buffered saline (PBS; Invitrogen; Thermo Fisher Scientific, Inc.), fixed with a mixture of methanol (MingRui Biotechnology Co., Ltd., Shanghai, China) and acetic acid (1:1 ratio; MingRui Biotechnology Co., Ltd.) and then stained with 1\% crystal violet (MingRui Biotechnology Co., Ltd.). The colonies containing $>50$ cells were counted under an inverted microscope (DMI3000B; Leica Microsystems GmbH, Wetzlar, Germany). The assay was performed in triplicate and repeated once.

Flow cytometric analysis of apoptosis. Tumor cell apoptosis was detected using flow cytometry using an Annexin V-fluorescein isothiocyanate (FITC) apoptosis 
A
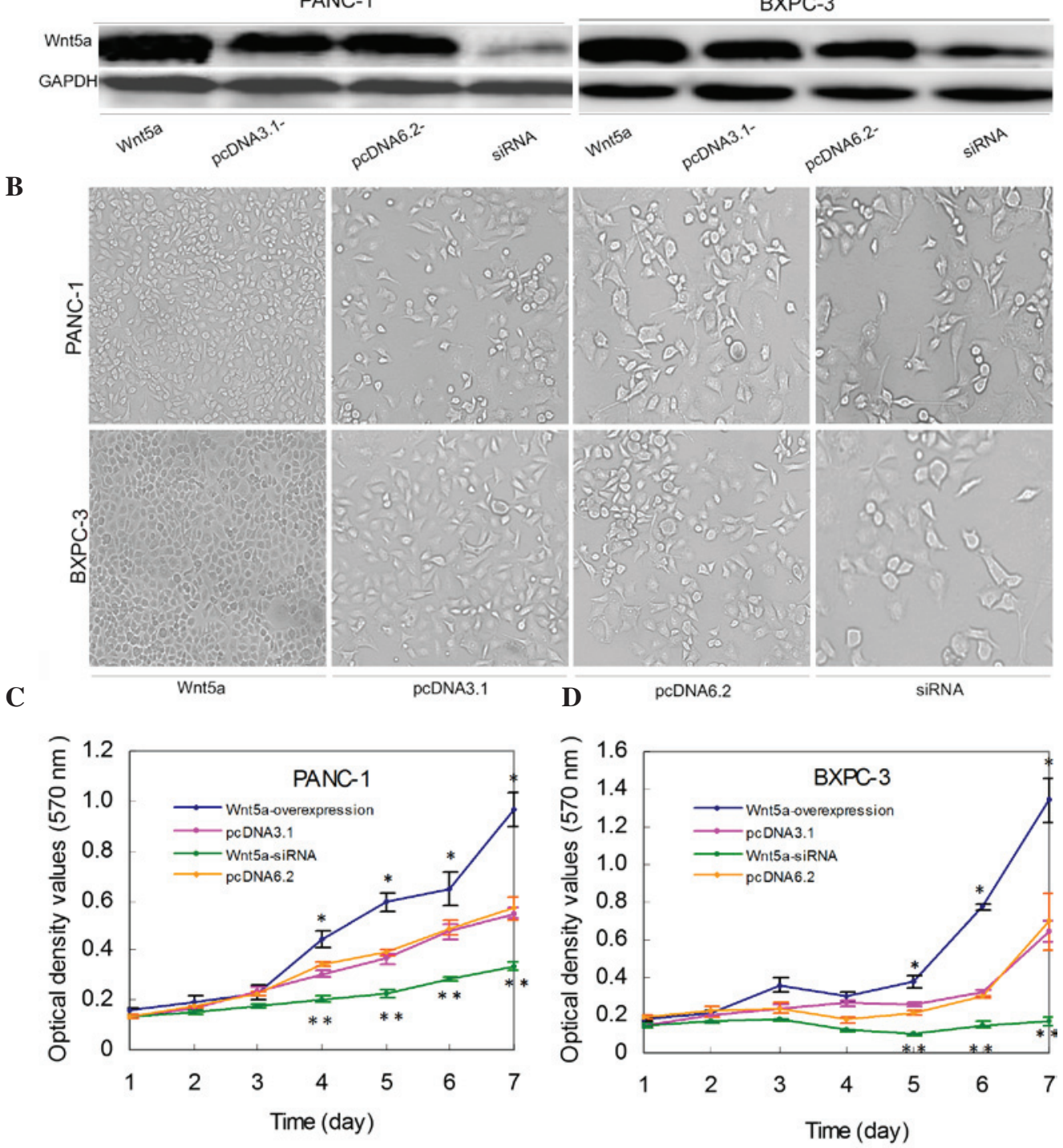

Figure 1. Wnt5a increases the viability of pancreatic cancer cells. (A) Western blot analysis of the protein expression of Wnt5a following Wnt5a pcDNA3.1, siRNA or pcDNA6.2 vectors being stably transfected into PANC-1 and BXPC-3 cells. GAPDH was used as an internal control. The experiments were repeated three times. (B) Monolayer cell culture. Representative images of the morphology of indicated cells following 7 days culture under a phase-contrast microscope. Magnification, x200. (C and D) Cell viability assay. The stably transfected cells were grown in a monolayer for up to 7 days and then subjected to a cell viability assay. siRNA, small interfering RNA. The values are presented as means \pm standard deviation. * P $<0.05$ of Wnt5a-overexpressing cells, vs. the control. ${ }^{* *} \mathrm{P}<0.05$ of Wnt5a-siRNA cells, vs. the control.

detection kit(cat.no.MR-KA020) from MingRuiBiotechnology Co. Ltd., according to the manufacturer's protocol. Briefly, near- $80 \%$ confluent PANC-1 and BXPC-3 cells were harvested using $0.25 \%$ trypsin (MingRui Biotechnology Co., Ltd.), washed twice in PBS and resuspended at a density of $1 \times 10^{6}$ viable cells/ml in Annexin $\mathrm{V}$ binding buffer (MingRui Biotechnology Co., Ltd.). Subsequently, $5 \mathrm{ml}$ Annexin V/FITC, at a final concentration of $50 \mu \mathrm{g} / \mathrm{ml}$, was added to the cell suspension with $10 \mu \mathrm{l}$ propidium iodide, at a final concentration of $1 \mu \mathrm{g} / \mathrm{ml}$. The cell solution was incubated for $15 \mathrm{~min}$ at room temperature in the dark. Following incubation, the stained cells were immediately analyzed using a flow cytometer (FACSCalibur; Bio-Rad Laboratories, Inc.). Each experiment was performed in triplicate and repeated at least three times.

Animal experiments. A total of 12 nude mice (nu/nu-nuBR), aged 4 weeks old and weighing 14-16 g, were obtained from the Shanghai Laboratory Animal Center, Chinese Academy of Science. The animals were housed in a specific pathogen-free environment with a constant temperature between 25 and $27^{\circ} \mathrm{C}$ in a 12/12 h light/dark cycle. The mice were fed with a standard pellet diet and water ad libitum. The present study was approved by the ethics committee of The Second Military Medical University (Shanghai, China) and was performed in compliance with the regulations of the Administration of Experimental Animals of the State Council in China.

The nude mice were anesthetized using $1 \%$ sodium pentobarbital (Shenggong Biotech Co., Ltd.) and surgery was performed to expose the pancreas and spleen. A total of $5 \times 10^{7}$ pancreatic cancer, PANC-1 or BXPC-3 cells, transfected with Wnt5a-overexpressing or empty vector, were injected into the pancreatic parenchyma, close to the hilum of the spleen. Subsequently, the pancreas was relocated into the abdominal 
A
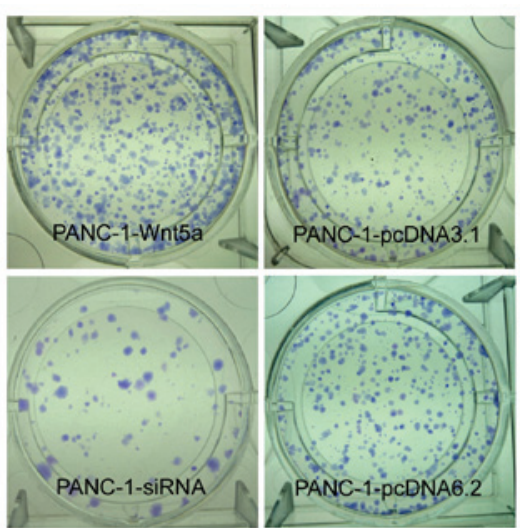

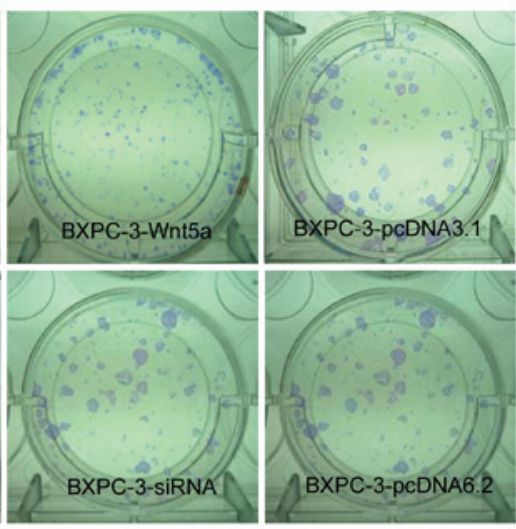

C 35
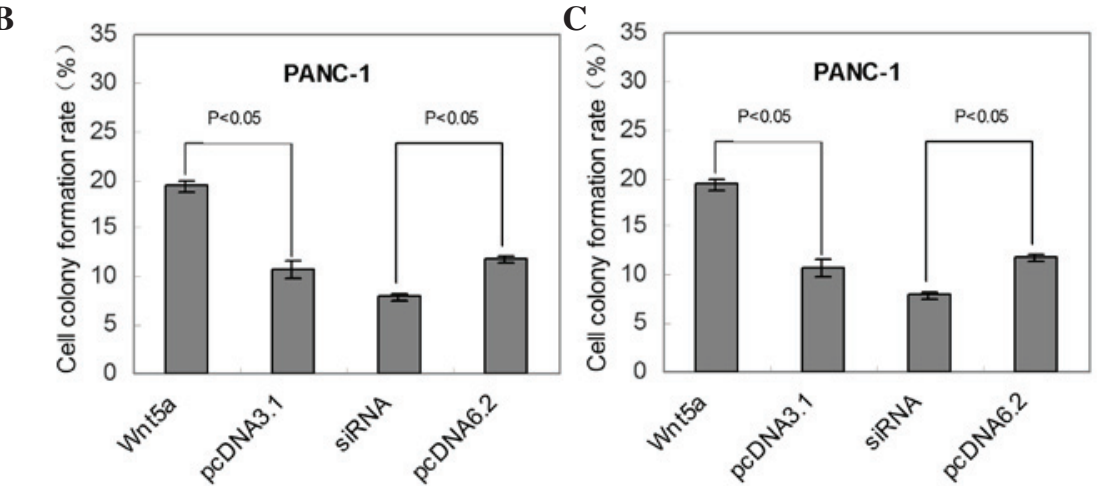

Figure 2. Wnt5a induces colony formation of PANC-1 and BXPC-3 cells. (A) Colony formation assay. Images of the cells in one of three independent experiments are shown. Graphs show the colony formation rates of the (B) PANC-1 cells and (C) BXPC-3 cells from three independent experiments. Data are expressed as the mean \pm standard error of the mean. siRNA, small interfering RNA.
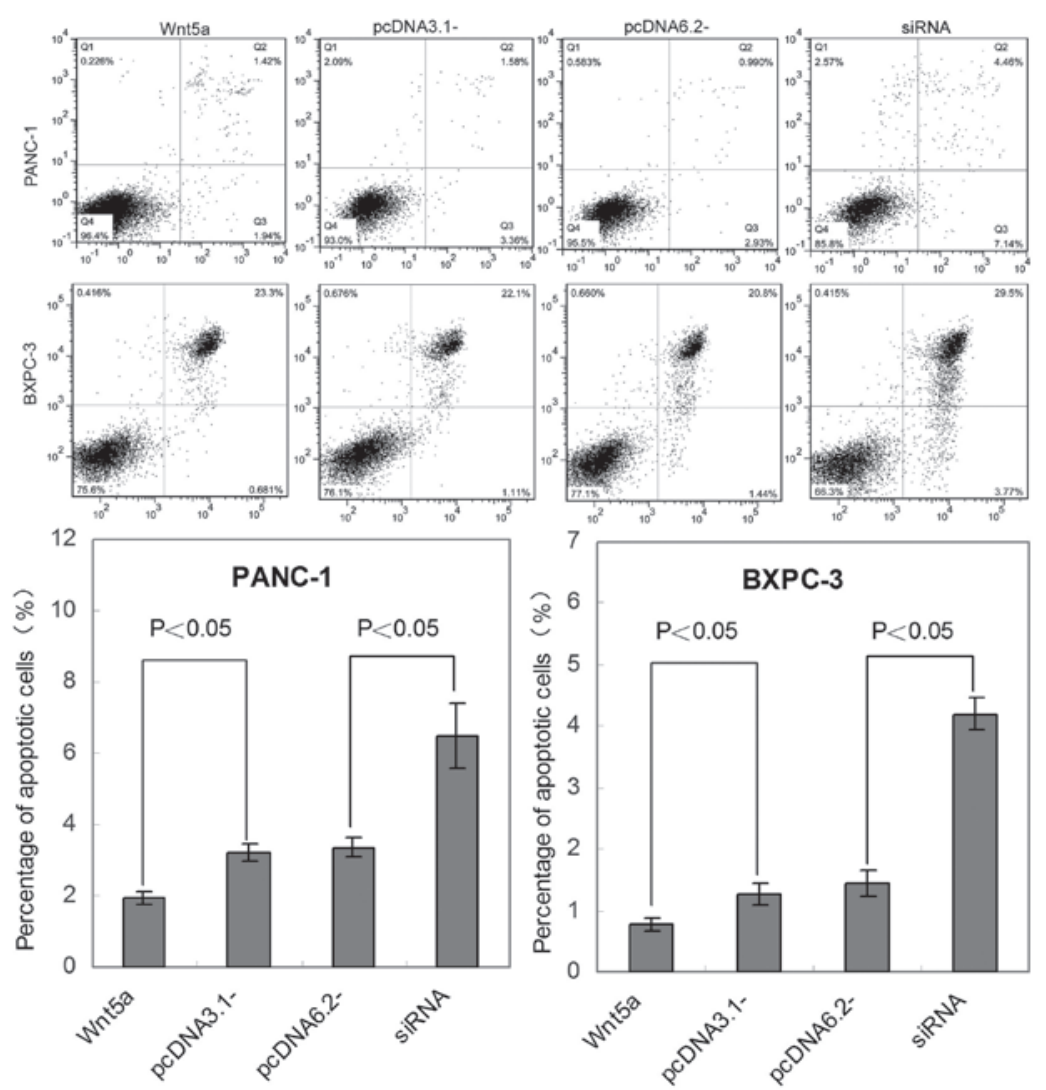

Figure 3. Overexpression of Wnt5a inhibits the apoptosis of PANC-1 and BXPC-3 cells. The percentages of Annexin V-positive cells were examined to evaluate the effect of Wnt5a on resistance to apoptosis in PANC-1 and BXPC-3 cells. Representative results of flow cytometric analysis are shown in the top panels. Q1 $\left(\mathrm{FITC}^{+} / \mathrm{PI}^{+}\right)$, cell debris; Q2 (FITC $\left./ \mathrm{PI}^{-}\right)$, necrosis cells; Q3 $\left(\mathrm{FITC}^{+} / \mathrm{PI}^{+}\right)$, apoptosis cells; Q4 (FITC $\left./ \mathrm{PI}^{-}\right)$, viable cells. The bar graphs show the quantitative results of cell apoptosis. Results are expressed as the mean \pm standard deviation. siRNA, small interfering RNA. 


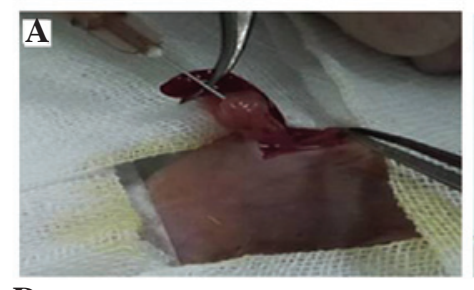

D

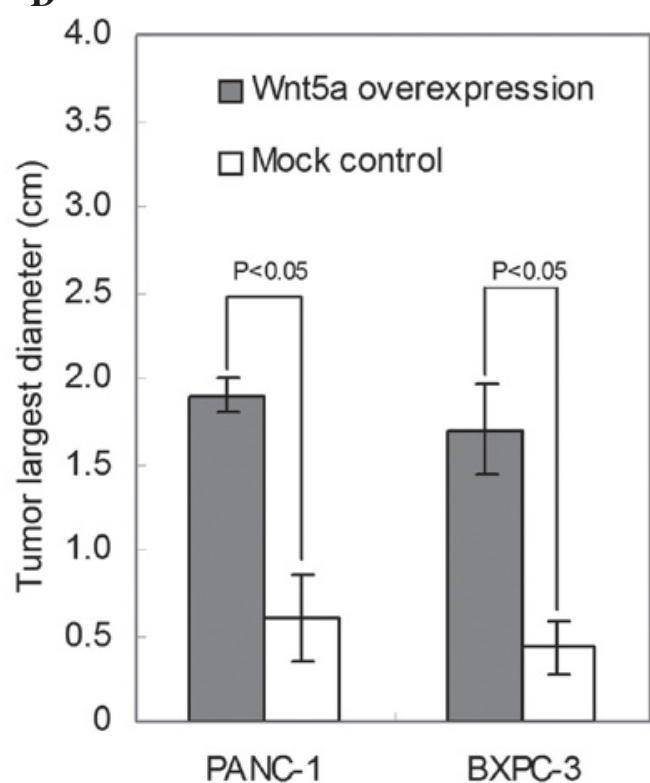

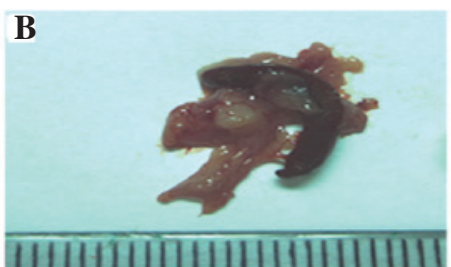

E

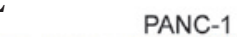

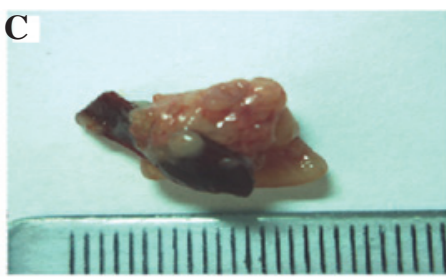

BXPC-3

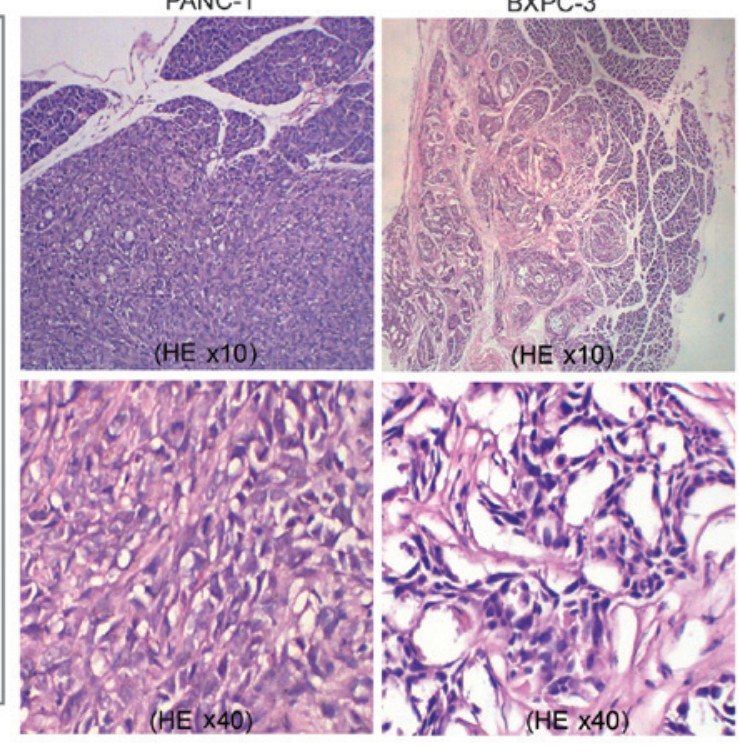

Figure 4. Wnt5a induces tumor growth in an orthotopic mouse model of pancreatic cancer cells. (A) Image of dissected pancreatic tissue from the mouse model and injection of pancreatic cancer cells into the pancreatic parenchyma. (B) Representative images of the implantation of tumor tissues in the Wnt5a-overexpressed PANC-1cells. (C) Representative images of implantation of tumor tissues of the control cells. (D) Bar graph shows the average diameters of the orthotopic tumors in each condition. Results are expressed as the mean \pm standard deviation. (E) HE staining. Representative micrographs show tumor morphology in the tumor tissues of the BXPC-3 and PCNA-1 cells. HE, hematoxylin and eosin.

cavity, which was then closed in two layers using 6-0 absorbable vicryl sutures [Anhui Kangning Industrial (Group) Co., Ltd, Shanghai, China]. The animals were divided into 4 groups, PANC-1-Wnt5a-overexpressing, PANC-1-mock control, BXPC-3-Wnt5a-overexpressing and BXPC-3-mock control, according to the different cells injected. Each group consisted of three animals. When the orthotopic tumors reached a size of $1-2 \mathrm{~cm}$ in diameter, the mice were sacrificed by exposure to $\mathrm{CO}_{2}$ followed by cervical dislocation. Images of the pancreatic tissues with tumor lesions were captured using a DSC-HX50 Sony camera (Sony Corporation, Tokyo, Japan), and the xenografts were resected, fixed in formalin (Shenggong Biotech Co., Ltd.), embedded in paraffin (Shenggong Biotech Co., Ltd.) and cut into $0.4 \mu \mathrm{m}$ tissue sections. A streptavidin-peroxidase-biotin immunohistochemical method was used for immunostaining. Briefly, tissue sections were deparaffinized and hydrated in xylene (ShengGong Biotechnology Co., Ltd.) and serial alcohol solutions (ShengGong Biotechnology Co., Ltd.). Endogenous peroxidase was blocked by incubation in $3 \% \mathrm{H}_{2} \mathrm{O}_{2}$ (ShengGong Biotechnology Co., Ltd.) for $15 \mathrm{~min}$ at room temperature. The antigen retrieval step was performed in a steam pressure cooker containing preheated $0.01 \mathrm{M}$ citrate buffer $(\mathrm{pH} 6.0$; Dako, Glostrup, Denmark) at $95^{\circ} \mathrm{C}$ for 15 min. Rabbit polyclonal anti-Wnt5a antibody (cat. no. ab72583; Abcam; 1:50) and rabbit polyclonal anti-Ki67 antibody (cat. no. ab15580;
Abcam; 1:100) were incubated with the tissue sections for $2 \mathrm{~h}$ at room temperature. The tissue sections were then treated with biotinylated polyclonal anti-rabbit secondary antibodies (cat. no. ab6720; Abcam; 1:1,000), followed by incubation with streptavidin-horseradish peroxidase complex (Santa Cruz Biotechnology, Inc.) for $30 \mathrm{~min}$ at room temperature. Immunoreactivity was visualized with diaminobenzidine (Sigma-Aldrich). The tissue sections were then counterstained with hematoxylin (ShengGong Biotechnology Co., Ltd.). Wnt5a staining was predominantly located in the cell cytoplasm and nuclei, whereas Ki67 staining in the nuclei. The proportion of positive tumor cells was scored.

Statistical analysis. Statistical analyses were performed using the SPSS 17 software package (SPSS, Inc., Chicago, IL, USA). Differences in the means were determined using Student's $t$-test or one-way analysis of variance followed by Tukey's post-hoc test. $\mathrm{P} \leq 0.05$ was considered to indicate a statistically significant difference.

\section{Results}

Wnt5a induces pancreatic cancer cell viability and colony formation. Stable Wnt5a-overexpressing or Wnt5a siRNA-expressing pancreatic cancer cell lines were established following gene transfection and confirmed using 


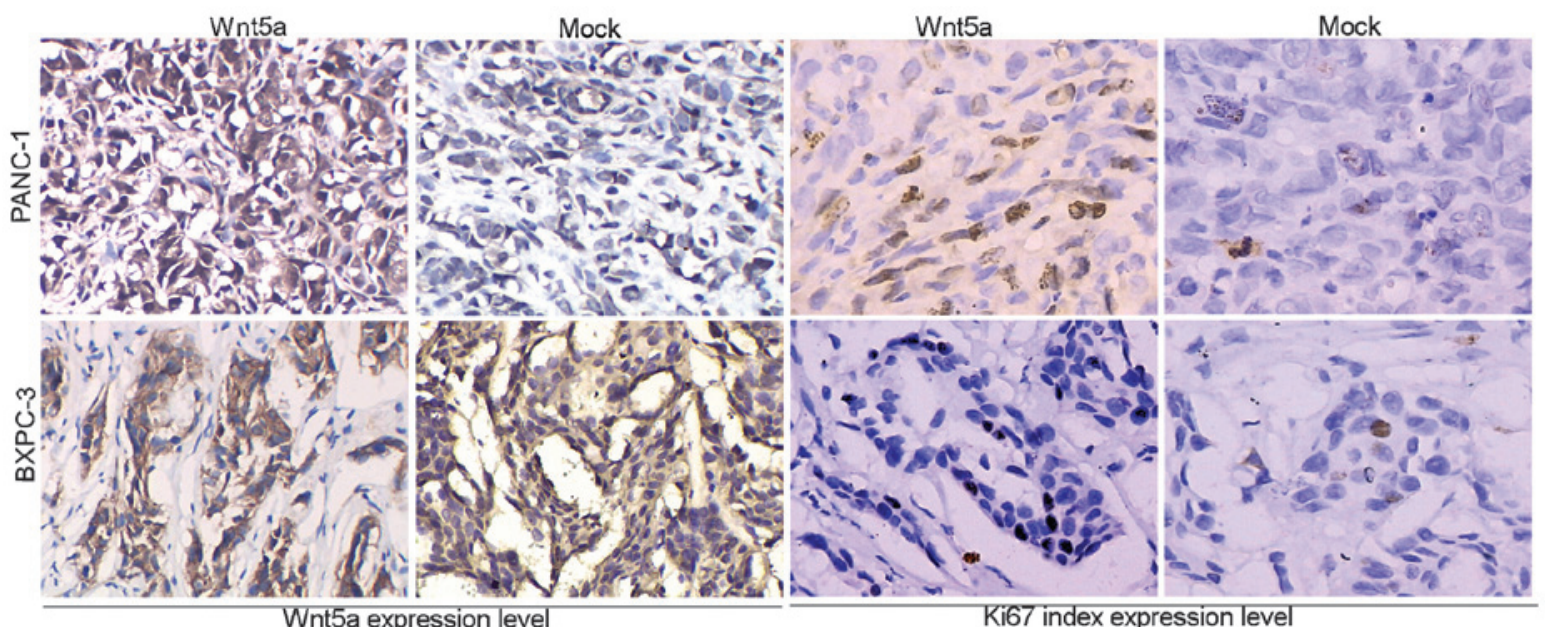

Figure 5. Immunohistochemical analysis. Tumor tissues from the control and Wnt5a-overexpressing BXPC-3 and PCNA-1 cells were immunostained with anti-Wnt5a or anti-Ki67 antibodies. Wnt5a was expressed in the cytoplasm and nuclei, whereas Ki67 was only expressed in the nuclei of the pancreatic cancer cells. Magnification, x400.

A

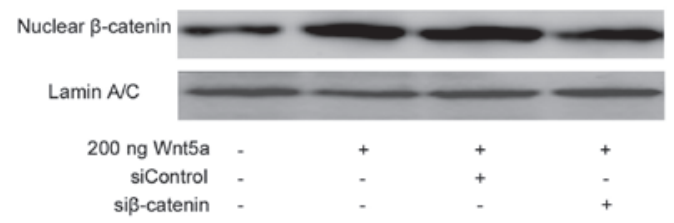

B

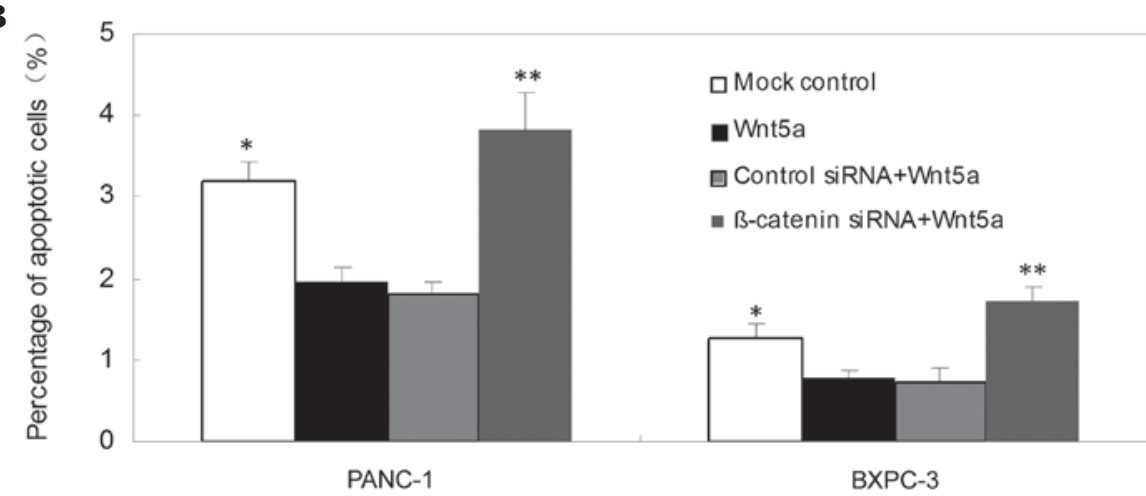

Figure 6. $\beta$-catenin mediates the effects of Wnt5a on pancreatic cancer cells. (A) Western blot analysis. Tumor cells were grown and transfected with $\beta$-catenin siRNA and then treated with recombinant Wnt5a protein prior to western blot analysis. (B) Flow cytometric assay of apoptosis. The cells were subjected to flow cytometric analysis to determine the percentage of apoptotic cells. The bar graph shows the results of densitometric analysis of the apoptotic assays. siRNA, small interfering RNA. The values are presented as the mean \pm standard deviation. ${ }^{*} \mathrm{P}<0.05$ of recombinant Wnt5a, vs. the control. ${ }^{* *} \mathrm{P}<0.05$ of $\beta-$ catenin siRNA, vs. the control.

immunoblot analysis (Fig. 1A). A subsequent cell viability assay showed that the cell density of PANC-1 and BXPC-3 cells was significantly different among the groups following seven days of culture (Fig. 1B). Statistical data confirmed that the numbers of viable cells were significantly higher in the Wnt5a-overexpressing cells, compared with that in the control cells $(\mathrm{P}<0.05$; Fig. 1B-D). By contrast, siRNA-mediated silencing of the expression of Wnt5a significantly reduced cell viability, compared with the control ( $\mathrm{P}<0.05$; Fig. 1B-D).

Furthermore, as shown in Fig.2A, the Wnt5a-overexpressing PANC-1 and BXPC-3 cells formed an increased number of colonies, compared with the control cells (211.9 and $180.7 \%$, respectively; $\mathrm{P}<0.05$; Fig. $2 \mathrm{~B}$ and $\mathrm{C}$ ). The siRNA transfected
Wnt5a PANC-1 and BXPC-3 cells exhibited a significant decrease in colony formation, compared with the control cells $(32.8 \%$ for PANC-1 and $35.77 \%$ for BXPC-3; $\mathrm{P}<0.05$; Fig. 2B and C).

Overexpression of Wnt5a inhibits tumor cell apoptosis. An Annexin V-FITC kit was used to further assess the effect of Wnt5a on pancreatic cancer apoptosis in vivo. Cells stained with Annexin $\mathrm{V}^{+} / \mathrm{PI}^{-}$(in Q3) are apoptotic. Compared with the control, the levels of apoptosis were significantly decreased in the Wnt5a-overexpressing PANC-1 and BXPC-3 cell lines $(\mathrm{P}<0.05)$, whereas an elevated rate of tumor cell apoptosis occurred in the siRNA-transfected tumor cells (Fig. 3). 
Wnt5a induces pancreatic cancer cell xenograft growth in an orthotopic nude mouse model. Following the in vitro investigations, the present study investigated the in vivo effects of Wnt5a on tumor growth using an orthotopic mouse model. The PANC-1 and BXPC-3 cells overexpressing Wnt5a were injected orthotopically into the pancreatic parenchyma, and empty vector-transfected cells were used as a control (Fig. 4A). The data showed that, consistent with the results of the in vitro assays, the average tumor size of the Wnt5a-overexpressing group was significantly larger, compared with that of the control group ( $\mathrm{P}<0.05$; Fig. 4B-D). Microscopic examination showed that PANC-1 and BXPC-3 cells exhibited different histological morphologies (Fig.4E). In addition, microscopic examination and immunohistochemistry analysis demonstrated that the Wnt5a-overexpressing tumors exhibited increased protein expression levels of Ki67 (Fig. 5).

$\beta$-catenin mediates the effects of Wnt5a on pancreatic cancer cells. To examine the molecular mechanisms mediating the effects of Wnt5a overexpression in pancreatic cancer cells, the present study pre-transfected $\beta$-catenin siRNA into pancreatic cancer cells, and then treated the cells with a recombinant Wnt5a peptide (200 ng/well). The data showed that the depletion of $\beta$-catenin reversed the effects of Wnt5a overexpression on the levels of apoptosis in the PANC-1 and BXPC-3 cells (Fig. 6A and B).

\section{Discussion}

Previous studies have demonstrated that Wnt5a can modulate various cell activities, including cell migration (18), adhesion (19), invasion (20) and differentiation (21). Specifically, Wnt5a promotes the progression of prostate and lung cancer cells $(22,23)$, however it is able to suppress tumor progression in thyroid carcinoma cells (24). In the present study, the effects of Wnt5a overexpression and knockdown on the proliferation of pancreatic cancer cells were examined. The results demonstrated that overexpression of the Wnt5a protein induced tumor cell viability and clonogenicity, but inhibited apoptosis in the two pancreatic cancer cell lines in vitro, and increased tumor growth in an orthotopic nude mouse model. By contrast, knockdown of the expression of Wnt5a reduced tumor cell viability and promoted apoptosis. At a molecular level, $\beta$-catenin mediated the effects of Wnt5a on the pancreatic cancer cells. The data from the present study indicated that Wnt5a is involved in the modulation of pancreatic cancer cell proliferation, and that Wnt5a may be a potential target for pancreatic cancer therapy.

PANC-1 and BXPC-3 cells are moderately to poorly differentiated pancreatic cancer cell lines with different morphological phenotypes and proliferative potential. When cultured in vitro, the PANC-1 cells were spindle-shaped and scattered in growth, whereas the BXPC-3 cells were arranged in nets with a polygonal shape. Despite their differences, the present study obtained consistent results following Wnt5a manipulation in vitro and in the orthotopic nude mouse model. A previous study by Ripka et al (25) demonstrated that transient knockdown of Wnt5a using hWNT5A-siRNA reduces the viability of the PANC-1 and MiaPaca2 pancreatic cancer cells, consistent with the data obtained in the present study. In addition, a clonogenicity assay was used in the present study to assess the group dependency of individual tumor cells and single cell proliferation capability in vitro. Several studies have indicated that Wnt5a has an effect on clonogenicity in different cancer cell lines. For example, Li et al showed that the ectopic expression of Wnt5a results in a significant inhibition of clonogenicity in esophageal squamous cell carcinoma cells (26), whereas Ying et al showed that transfection of a dominant-negative Wnt5a, a nonfunctional short isoform with the WNT domain deleted, results in substantial inhibition of colorectal cancer cell clonogenicity (27) and K562 tumor cell clonogenicity (28). However, Kremenevskaja et al showed that transfection of Wnt5a in a FTC-133 thyroid tumor cell line reduces cell clonogenicity (24). The results of these previous studies indicate that Wnt5a is tumor type-specific and/or dependent on the whole Wnt5a gene pathway, and may not be just a Wnt5a protein. In the present study, the data showed that pancreatic cancer cell lines overexpressing Wnt5a protein enhanced clonogenicity, whereas suppression of Wnt5a reduced clonogenicity.

Furthermore, the role of Wnt5a in cell apoptosis has been shown to be controversial in previous reports and appears to be cell type-specific. Several studies have indicated that Wnt5a has anti-apoptotic actions in different cell lines. For example, the expression of Wnt5a inhibits serum starvation-induced apoptosis in primarily cultured human dermal fibroblasts (29), HEK293 cells (30), MC3T3-E1 osteoblast cells (31) and isolated germinal center B cells (32). In addition, Vuga et al showed that the expression of $\mathrm{Wnt} 5 \mathrm{a}$ induces resistance to $\mathrm{H}_{2} \mathrm{O}_{2}$-induced apoptosis in fibroblasts isolated from lung tissues with interstitial pneumonia (33). However, additional studies have reported that Wnt5a has a pro-apoptotic action. Liang et al showed that loss of Wnt5a inhibits the apoptosis of $\mathrm{CD}^{+} / \mathrm{CD}^{+}$thymocytes, whereas exogenous Wnt5a increases the apoptosis of fetal thymocytes in vitro (34). Peng et al (35) showed that Wnt5a markedly increases serum starvation-induced apoptosis in placental choriocarcinoma cells, compared with untransfected control cells. In the present study, the results revealed that overexpression of the Wnt5a protein promoted tumor cell survival/resistance to apoptosis in the pancreatic cancer cell lines, whereas downregulation of the Wnt5a protein promoted cell apoptosis. These data indicated a possible effect of Wnt5a on the regulation of survival and resistance to apoptosis in pancreatic cancer cells. Furthermore, the data obtained using the orthotopic nude mouse model showed that the overexpression of Wnt5a promoted tumor growth and increased the protein expression of Ki67 in tumor xenografts. Taken together, these data indicate that Wnt5a exerted distinct effects on the pancreatic cancer cells through the promotion of tumor growth and the inhibition of tumor cell apoptosis. As such, it acts as an oncogene in pancreatic cancer.

Although Wnt5a, as a non-canonical Wnt, is involved in almost all aspects of the non-canonical Wnt signaling pathway, previous studies have shown that Wnt5a is able to activate $\beta$-catenin signaling, based on specific receptor availability (36). Wnt5a is able to switch between two considerably diverse forms of signaling $(37,38)$. Increasing evidence indicates a critical role for the Wnt5a- $\beta$-catenin signaling pathway in the pathogenesis of pancreatic cancer $(39,40)$. It has been reported that the addition of recombinant Wnt5a leads to increased nuclear 
$\beta$-catenin in PANC-1 cells, as well as a significant increase in T-cell factor/lymphoid enhancer factor-dependent reporter activity (25), indicating the activation of the canonical signaling pathway. Wang et al (39) also documented that expression of the ataxia-telangiectasia group D complementing gene increases the levels of $\beta$-catenin in pancreatic cancer, consequently facilitating tumor growth and metastasis. In our previous study, data revealed the promotion of $\beta$-catenin nuclear translocation in PANC-1 and BXPC-3 cells by exposure to recombinant Wnt5a (17). In the present study, it was observed that the depletion of $\beta$-catenin reversed the effects of the recombinant Wnt5a protein on the apoptosis of pancreatic cancer cells. This indicated that the Wnt5a- $\beta$-catenin canonical pathway was involved in pancreatic cancer cell behavior and in the progression of pancreatic cancer.

The data presented in the present study provide significant support for a role for Wnt5a in the development of pancreatic cancer, and are consistent with our previous findings, which showed altered expression of Wnt5a in pancreatic cancer tissues and an association of Wnt5a with certain clinicopathological data (17). However, the results of the present study can be considered as proof-of-principle, and further investigation is required to clarify the role of Wnt5a in pancreatic cancer. Future investigations aim to further elucidate the molecular mechanism underlying the effects of Wnt5a in pancreatic cancer.

\section{Acknowledgements}

This study was supported by grants from the National Key Project of Scientific and Technical Supporting Programs of China (grant no, 2006BAI02A14) and the National Natural Science Foundation of China (grant nos. 30770996 and 81172310) to Dr Minghua Zhu.

\section{References}

1. Pandol S, Gukovskaya A, Edderkaoui M, Dawson D, Eibl G and Lugea A: Epidemiology, risk factors and the promotion of pancreatic cancer: Role of the stellate cell. J Gastroenterol Hepatol 27 (Suppl 2): S127-S134, 2012.

2. Siegel R, Ma J, Zou Z and Jemal A: Cancer statistics, 2014. CA Cancer J Clin 64: 9-29, 2014.

3. Vincent A, Herman J, Schulick R, Hruban RH and Goggins M: Pancreatic cancer. Lancet 378: 607-620, 2011.

4. Maitra A, Kern SE and Hruban RH: Molecular pathogenesis of pancreatic cancer. Best Pract Res Clin Gastroenterol 20: 211-226, 2006.

5. Moore PS, Sipos B, Orlandini S, Sorio C, Real FX, Lemoine NR, Gress T, Bassi C, Klöppel G, Kalthoff $\mathrm{H}$, et al: Genetic profile of 22 pancreatic carcinoma cell lines. Analysis of K-ras, p53, p16 and DPC4/Smad4. Virchows Arch 439: 798-802, 2001.

6. Li D, Xie K, Wolff R and Abbruzzese JL: Pancreatic cancer. Lancet 363: 1049-1057, 2004.

7. Logan CY and Nusse R: The Wnt signaling pathway in development and disease. Annu Rev Cell Dev Biol 20:781-810, 2004.

8. Qian D, Jones C, Rzadzinska A, Mark S, Zhang X, Steel K, Dai X and Chen P: Wnt5a functions in planar cell polarity regulation in mice. Dev Biol 306: 121-133, 2007.

9. Clevers $\mathrm{H}$ : Wnt/beta-catenin signaling in development and disease. Cell 127: 469-480, 2006

10. Wong GT, Gavin BJ and McMahon AP: Differential transformation of mammary epithelial cells by Wnt genes. Mol Cell Biol 14: 6278-6286, 1994.

11. Fang DR, Lv ZF and Qiao G: Dynamic Wnt5a expression in murine hair follicle cycle and its inhibitory effects on follicular. Asian Pac J Trop Med 7: 285-288, 2014.
12. Maeda K, Kobayashi Y, Udagawa N, Uehara S, Ishihara A, Mizoguchi T, Kikuchi Y, Takada I, Kato S, Kani S, et al: Wnt5a-Ror2 signaling between osteoblast-lineage cells and osteoclast precursors enhances osteoclastogenesis. Nat Med 18: 405-412, 2012

13. Christman MA 2nd, Goetz DJ, Dickerson E, McCall KD, Lewis CJ, Benencia F, Silver MJ, Kohn LD and Malgor R: Wnt5a is expressed in murine and human atherosclerotic lesions. Am J Physiol Heart Circ Physiol 294: 2864-2870, 2008.

14. BakkerER, Das AM, Helvensteijn W, Franken PF, Swagemakers S, van der Valk MA, ten Hagen TL, Kuipers EJ, van Veelen W and Smits R: Wnt5a promotes human colon cancer cell migration and invasion but does not augment intestinal tumorigenesis in Apc1638N mice. Carcinogenesis 34: 2629-2638, 2013.

15. Trifa F, Karray-Chouayekh S, Jmal E, Jmaa ZB, Khabir A, Sellami-Boudawara T, Frikha M, Daoud J and Mokdad-Gargouri R: Loss of WIF-1 and Wnt5a expression is related to aggressiveness of sporadic breast cancer in Tunisian patients. Tumour Biol 34: 1625-1633, 2013.

16. Lee SE, Lim SD, Kang SY, Suh SB and Suh YL: Prognostic significance of Ror2 and Wnt5a expression in medulloblastoma. Brain Pathology 23: 445-453, 2013.

17. Bo H, Zhang S, Gao L, Chen Y, Zhang J, Chang X and Zhu M: Upregulation of Wnt5a promotes epithelial- to mesenchymal transition and metastasis of pancreatic cancer cells. BMC Cancer 13: 496, 2013.

18. Nishita M, Yoo SK, Nomachi A, Kani S, Sougawa N, Ohta Y, Takada S, Kikuchi A and Minami Y: Filopodia formation mediated by receptor tyrosine kinase Ror2 is required for Wnt5a-induced cell migration. J Cell Biol 175: 555-562, 2006.

19. Matsumoto S, Fumoto K, Okamoto T, Kaibuchi K and Kikuchi A: Binding of APC and dishevelled mediates Wnt5a-regulated focal adhesion dynamics in migrating cells. EMBO J 29: 1192-1204, 2010.

20. Yamamoto H, Kitadai Y, Yamamoto H, Oue N, Ohdan H, Yasui W and Kikuchi A: Laminin gamma2 mediates Wnt5a-induced invasion of gastric cancer cells. Gastroenterology 137: 242-252, 2009.

21. Lehtonen A, Ahlfors H, Veckman V, Miettinen M, Lahesmaa R and Julkunen I: Gene expression profiling during differentiation of human monocytes to macrophages or dendritic cells. J Leukoc Biol 82: 710-720, 2007.

22. Yamamoto H, Oue N, Sato A, Hasegawa Y, Yamamoto $H$, Matsubara A, Yasui W and Kikuchi A: Wnt5a signaling is involved in the aggressiveness of prostate cancer and expression of metalloproteinase. Oncogene 29: 2036-2046, 2010.

23. Huang CL, Liu D, Nakano J, Ishikawa S, Kontani K, Yokomise H and Ueno M: Wnt5a expression is associated with the tumor proliferation and the stromal vascular endothelial growth factor-an expression in non-small-cell lung cancer. J Clin Oncol 23: 8765-8773, 2005.

24. Kremenevskaja N, von Wasielewski R, Rao AS, Schöfl C, Andersson T and Brabant G: Wnt-5a has tumor suppressor activity in thyroid carcinoma. Oncogene 24: 2144-2154, 2005.

25. Ripka S, Konig A, Buchholz M, Wagner M, Sipos B, Kloppel G, Downward J, Gress T and Michl P: WNT5A-target of CUTL1 and potent modulator of tumor cell migration and invasion in pancreatic cancer. Carcinogenesis 28: 1178-1187, 2007.

26. Li J, Ying J, Fan Y, Wu L, Ying Y, Chan AT, Srivastava G and Tao Q: WNT5A antagonizes WNT/ $\beta$-catenin signaling and is frequently silenced by promoter $\mathrm{CpG}$ methylation in esophageal squamous cell carcinoma. Cancer Biol Ther 10: 617-624, 2010.

27. Ying J, Li H, Yu J, Ng KM, Poon FF, Wong SC, Chan AT, Sung JJ and Tao Q: WNT5A exhibits tumor-suppressive activity through antagonizing the Wnt/beta-catenin signaling, and is frequently methylated in colorectal cancer. Clin Cancer Res 14: 55-61, 2008.

28. Ying J, Li H, Chen YW, Srivastava G, Gao Z and Tao Q: WNT5A is epigenetically silenced in hematologic malignancies and inhibits leukemia cell growth as a tumor suppressor. Blood 110: 4130-4131, 2007.

29. Torii K, Nishizawa K, Kawasaki A, Yamashita Y, Katada M, Ito M, Nishimoto I, Terashita K, Aiso S and Matsuoka M: Anti-apoptotic action of Wnt5a in dermal fibroblasts is mediated by the PKA signaling pathways. Cell Signal 20: 1256-1266, 2008.

30. Jia L, Miao C, Cao Y and Duan EK: Effects of Wnt proteins on cell proliferation and apoptosis in HEK293 cells. Cell Biol Int 32: 807-813, 2008. 
31. Almeida M, Han L, Bellido T, Manolagas SC and Kousteni S: Wnt proteins prevent apoptosis of both uncommitted osteoblast progenitors and differentiated osteoblasts by beta-catenin-dependent and -independent signaling cascades involving Src/ERK and phosphatidylinositol 3-kinase/AKT J Biol Chem 280: 41342-41351, 2005.

32. Kim J, Kim DW, Chang W, Choe J, Kim J, Park CS, Song K and Lee I: Wnt5a is secreted by follicular dendritic cells to protect germinal center $\mathrm{B}$ cells via Wnt/Ca2+/NFAT/NF- $\kappa \mathrm{B}-\mathrm{B}$ cell lymphoma 6 signaling. J Immunol 188: 182-189, 2012.

33. Vuga LJ, Ben-Yehudah A, Kovkarova-Naumovski E, Oriss T, Gibson KF, Feghali-Bostwick C and Kaminski N: WNT5A is a regulator of fibroblast proliferation and resistance to apoptosis. Am J Respir Cell Mol Biol 41: 583-589, 2009.

34. Liang H, Coles AH, Zhu Z, Zayas J, Jurecic R, Kang J and Jones SN: Noncanonical Wnt signaling promotes apoptosis in thymocyte development. J Exp Med 204: 3077-3084, 2007.

35. Peng S, Zhang J, Chen J and Wang H: Effects of Wnt5a protein on proliferation and apoptosis in JAR choriocarcinoma cells. Mol Med Report 4: 99-104, 2011.
36. Umbhauer M, Djiane A, Goisset C, Penzo-Méndez A, Riou JF, Boucaut JC and Shi DL: The C-terminal cytoplasmic Lys-thr-X-X-X-Trp motif in frizzled receptors mediates Wnt/beta-catenin signaling. EMBO J 19: 4944-4954, 2000.

37. Mikels AJ and Nusse R: Purified Wnt5a protein activates or inhibits beta-catenin-TCF signaling depending on receptor context. PLoS Biol 4: e115, 2006.

38. van Amerongen R, Fuerer C, Mizutani M and Nusse R: Wnt5a can both activate and repress Wnt/ $\beta$-catenin signaling during mouse embryonic development. Dev Biol 369: 101-114, 2012.

39. Wang L, Heidt DG, Lee CJ, Yang H, Logsdon CD, Zhang L, Fearon ER, Ljungman $M$ and Simeone DM: Oncogenic function of ATDC in pancreatic cancer through Wnt pathway activation and beta-catenin stabilization. Cancer Cell 15: 207-219, 2009.

40. Kobayashi T, Shimura T, Yajima T, Kubo N, Araki K, Tsutsumi S, Suzuki H, Kuwano H and Raz A: Transient gene silencing of galectin-3 suppresses pancreatic cancer cell migration and invasion through degradation of $\beta$-catenin. Int J Cancer 129: 2775-2786, 2011 